\title{
THE MELANIZING ACTIVITY OF THE SEMEN OF BUFFALO BULLS AND ITS RELATION TO THE PROPORTION OF LIVE SPERMATOZOA
}

\author{
K. P. PANT* AND D. P. MUKHERJEE \\ Division of Animal Genetics, Indian Veterinary Research Institute, \\ Izatnagar, India
}

(Received 10th August 1970, revised 19th November 1970)

Melanizing activity of rabbit semen was reported by Beatty (1956). The activity - colour reaction when semen is supplied with dihydroxyphenylalaninevaried between males with different pelt colours: black rabbits showed intense melanizing activity and albinos none. It was further observed that the pale colour of semen was a characteristic of dark pelted bucks, and that the colour of the semen was, in some way, related to its melanizing activity. Mukherjee (1964) studied the melanizing activity of the semen of bulls, goats and rams, and observed that the activity varied between animals and between ejaculates from the same animal. Although there was no appreciable relationship between the coat colour of these animals and the melanizing activity of the semen, the yellow semen samples showed stronger melanizing activity than the non-yellow ones. The proportion of 'live' (unstained in eosin-nigrosin) spermatozoa was uniformly less in strongly melanizing semen samples than in weakly melanizing ones. In the present experiment, an attempt was made to study the melanizing activity of buffalo semen and its relationship to the proportion of 'live' spermatozoa.

For the experiment, twenty-two buffalow bulls of Murrah breed were available. All the bulls were jet black in colour, and were in regular use for artificial insemination. In all, fifty-four semen samples (four from each of two bulls, three from each of thirteen bulls, and one from each of the remaining seven bulls) were collected by means of an artificial vagina and tested for melanizing activity. The procedure for testing melanizing activity was same as that described by Mukherjee (1964) for bulls, and the colour reaction was graded according to the scale described by Beatty (1956).

Only two bulls (Nos. 530 and 372) were found to be positive for melanizing activity. The semen samples from these two bulls and from four other bulls (Nos. 399, 73, 471 and 405), which showed no melanizing activity and were randomly selected, were used to study the effect of various levels of cold shock on the proportion of 'live' spermatozoa. For this purpose, four semen samples were collected from each of the six bulls on different days (a total of twentyfour semen samples). From each sample, immediately after collection, a part was withdrawn and tested for melanizing activity. The remaining part was

\footnotetext{
* Present address: Institute of Animal Genetics, West Mains Road, Edinburgh EH9 3JN, Scotiand.
} 
held in a water bath at $33 \pm 1^{\circ} \mathrm{C}$. The effect of various levels of cold shock was studied according to the procedure described by Mukherjee (1964).

In both the groups (melanizing and non-melanizing) of semen samples, the mean percentage of 'live' spermatozoa decreased with increasing difference in the temperature of semen and the stain. The mean percentage was less in the

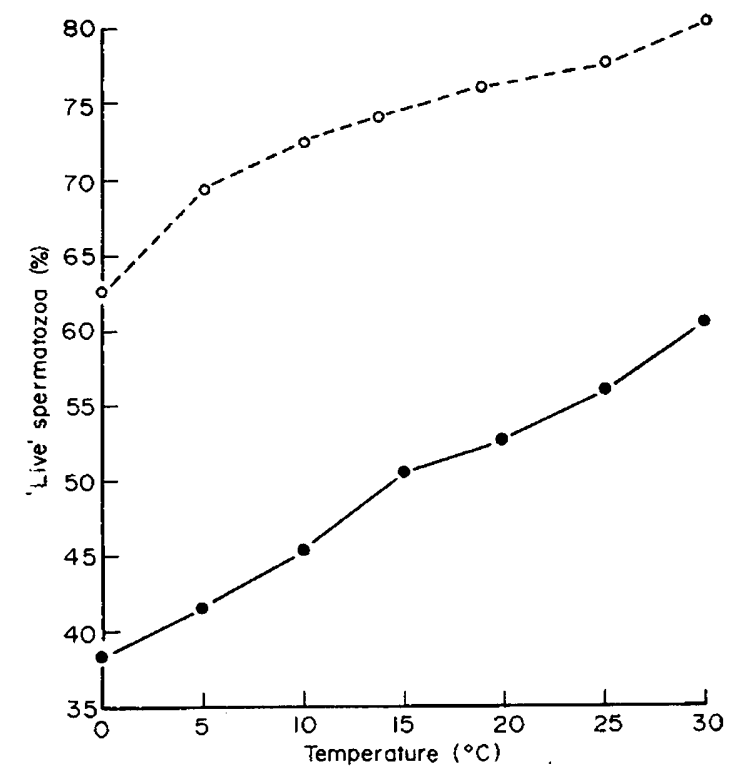

Text-Fig. 1. Percentage 'live' spermatozoa of buffalo bulls at various levels of temperature shock. $O$, Non-melanizing; $\bullet$, melanizing.

TABLE 1

ANALYSIS OF VARIANCE OF 'LIVE' BUFFALO SPERMATOZOA

\begin{tabular}{l|c|c}
\hline \multicolumn{1}{c|}{ Source of variation } & $\begin{array}{c}\text { Degrees of } \\
\text { freedom }\end{array}$ & Mean squares \\
\hline Groups & 1 & $21640 \cdot 2^{* * * *}$ \\
Bulls within groups & 4 & $8175 \cdot 5^{* * * *}$ \\
Semen samples within bulls & 18 & $275 \cdot 7 * * * *$ \\
Treatments & 6 & $1041 \cdot 6^{* * * *}$ \\
Groups $\times$ treatments & 6 & $22 \cdot 9^{*}$ \\
Bulls $\times$ treatments & 24 & $34 \cdot 6^{* * * *}$ \\
Error & 109 & $10 \cdot 0$ \\
& & \\
\hline \multicolumn{1}{c}{$* * * * P<0 \cdot 005}$. & $* 0.025<P<0 \cdot 05$.
\end{tabular}

melanizing than in the non-melanizing group at all levels of temperature shock (Text-fig. 1). The difference observed in the percentage of live spermatozoa was subjected to analysis of variance (Table 1). The mean square for groups (tested against the mean square for samples within groups) was highly significant. As the interactions were significant, the composite mean square for treatment and for error and their respective degrees of freedom were calculated 
(Johnson \& Keeping, 1952), and tested for significance. The variation due to treatments was highly significant.

The intensity of colour reaction varied between gradings of + and ++ . In bulls, goats and rams also, the colour reaction varied between similar gradings (Mukherjee, 1964) though in black or nearly black rabbits, it may be as high as ++++ (Beatty, 1956). As the buffalo bulls used in the present experiment were of uniform black colour and the melanizing activity in their semen was weaker than that reported in dark pelted rabbits (Beatty, 1956), it appears that there is no relationship between the melanizing activity and the coat colour of buffalo bulls. Of the two bulls showing melanizing activity, the semen of one (No. 530) was yellow in colour.

The trend of variations in the percentage of 'live' spermatozoa after various levels of temperature shock appeared linear in the two groups and not curvilinear as reported by Mukherjee (1964) in bulls, goats and rams.

The percentage of 'live' spermatozoa is directly related to the metabolic activity of semen and indirectly to the fertility and resistance to cold shock of spermatozoa (Bishop \& Hancock, 1955). The melanizing activity of the semen, therefore, may be worth considering when buffalo bulls are selected for artificial insemination, or for a study of the metabolic activity and the resistance to cold shock of the spermatozoa.

One of us (K.P.P.) is grateful to the Indian Council of Agricultural Research, New Delhi, for financial assistance.

\section{REFERENCES}

Beatty, R. A. (1956) Melanizing activity of semen from rabbit males of different genotypes. Proc. $R$. Soc. Edinb. B, 25, 39.

Bishop, M. W. H. \& Hancock, J. L. (1955) The evaluation of bull semen. Vet. Rec. 67, 363.

Johnson, L. P. V. \& Keeping, E. S. (1952) Composite mean squares and their degrees of freedom. Applied Statistics, 1, 202. (cf. Jornson, L. P. V. (1963) Biometrical methods, Institute for the Advancement of Science and Culture, New Delhi, p. 143.)

Mukherjee, D. P. (1964) Melanizing activity of semen and its relation to live spermatozoa of bulls, goats and rams. 7. Reprod. Fert. 7, 29. 